\title{
Steps in the formation of neurites and synapses studied in cultured leech neurons
}

\section{F.F. De-Miguel}

\author{
Correspondence \\ F.F. De-Miguel \\ Departamento de Biofísica \\ Instituto de Fisiología Celular \\ Universidad Nacional Autónoma \\ de México \\ Apartado Postal 70-253 \\ México, 04510 DF \\ México \\ Fax: + 52-5-622-5607 \\ E-mail: ffernand@ ifisiol.unam.mx \\ Presented at the International \\ Neurobiology Course, Faculdade \\ de 0 dontologia de Ribeirão Preto, \\ Universidade de São Paulo, Ribeirão \\ Preto, SP, Brasil, May 20-27, 1998. \\ Research supported by CONACYT, \\ PAPIIT and the Human Frontiers \\ Science Program to F.F. De-Miguel.

Departamento de Biofísica, Instituto de Fisiología Celular, Universidad Nacional Autónoma de México, DF, México

\section{Abstract}

Leech neurons in culture have provided novel insights into the steps in the formation of neurite outgrowth patterns, target recognition and synapse formation. Identified adult neurons from the central nervous system of the leech can be removed individually and plated in culture under well-controlled conditions, where they retain their characteristic physiological properties, grow neurites and form specific chemical or electrical synapses. Different identified neurons develop distinctive outgrowth patterns that depend on their identities and on the molecular composition of the substrate. On native substrates, the patterns displayed by these neurons reproduce characteristics from the adult or the developing neurons. In addition, the substrate may induce selective directed growth between pairs of neurons that normally make contact in the ganglion. Upon contact, pairs of cultured leech neurons form chemical or electrical synapses, or both types depending on the neuronal identities. Anterograde and retrograde signals during membrane contact and synapse formation modify the distribution of synaptic terminals, calcium currents, and responses to 5-hydroxytryptamine.

\section{Introduction}

During development and regeneration of the nervous system neurons must grow, find their targets and form the correct connections. Cultured neurons from adult invertebrates have been useful to characterize the steps from neurite outgrowth to synapse formation (1-5). After isolation, many invertebrate neurons remain differentiated and continue synthesizing the same transmitters, ionic channels and transmitter receptors as they normally do in the animal $(4,5)$. Moreover, pairs or triplets of neurons plated together regrow, recognize each other and form specific synapses (4-6). This review focuses on studies with cultured leech neurons, in which many of the steps from neurite outgrowth to synapse formation have been elucidated.

Identified neurons from adult leeches can be isolated one by one from the central nervous system (CNS) and kept in culture, where they regenerate neurites and synapses with different targets, allowing the study of these events step by step with single cell resolution (4-8). Different neurons can be identified visually in a very reliable way by their characteristic sizes and locations in the ganglion. After opening the ganglion capsule and incubating with proteolytic enzymes, individual neurons can be removed by applying suction through a glass pipette, sterilized and plated in culture (9). This procedure permits the experimenter to obtain cells with processes 
of variable length from which they will regrow and form synaptic connections with other neurons $(4,10)$. Early experiments showed that cultured leech neurons survive for weeks, preserving their resting potentials and the distinctive shape and duration of their action potentials (11). Moreover, cells can regrow neurites displaying characteristic branching patterns that depend on their identities and on the substrate $(7,8)$, and, when plated in contact with other cells, they form specific chemical or electrical synapses. Cell-to-cell contact and synapse formation induce changes in the distribution of release sites, ion channels and postsynaptic receptors.

\section{Sprouting and retracting neurites}

Neurite outgrowth during development and regeneration of the nervous system is regulated by growth-promoting molecules, soluble factors and cell-cell interactions (12). The family of extracellular matrix (ECM) proteins provides important sources for neurite growth, retraction and branching of neurites in the CNS as well as in culture (13). Different ECM proteins such as laminin, tenascin and fibronectin have been identified, isolated and cloned, and some of their functions regulating neurite extension or repulsion are known (12). Nevertheless, although studies analyzing attachment of neurons to purified molecules have provided extensive information as to some of the effects of these molecules, in the CNS of vertebrates and invertebrates ECM has a broad molecular composition, thus suggesting a complex regulation of neurite outgrowth. On the other hand, cell-cell interactions and soluble factors influence neurite outgrowth in embryos or regenerating nervous systems. How far could native ECM shape a neuronal outgrowth pattern in the absence of soluble factors and cell-cell interactions? The following studies, conducted on two different leech neurons plated on different substrates, have provided interesting insights as to how complex native mixtures of ECM proteins regulate neurite extension and directed growth.

Retzius neurons, which in the animal coordinate swimming, and anterior pagoda (AP) neurons, of unknown function, start growing a few minutes after being plated in culture with characteristic patterns that depend on the substrate and on the neuronal type. For each cell type the outgrowth patterns are consistently very similar, but quite different from patterns of the same cells on different substrates (14). An interesting example has been provided by AP or Retzius neurons plated on a substrate they normally contact in the animal, i.e., the ganglion capsule, which is enriched in ECM proteins. AP neurons plated on capsules (Figure 1A) develop a T-shaped pattern consisting of two main branches in opposite directions that later bifurcate $(15,16)$. A completely different pattern is developed by Retzius neurons plated on the same substrate, where they sprout a bundle of long and thick processes emerging from the axonal stump (Figure 1B). Plating a particular cell type on different substrates can be used to demonstrate the importance of the composition of the substrate in the outgrowth pattern. As can also be seen in Figure 1, the pattern of AP neurons on ganglion capsules is quite different from the patterns developed by the same neuronal type on other substrates. For example, AP neurons plated on leech laminin extracts extend long neurites with few branching points (Figure 1C). In contrast, on the plant lectin concanavalin A (Con A) (a substrate they never encounter in the animal), AP neurons extend flat and curved processes with multiple branching points (Figure 1D; 14,15).

In contrast to the pattern of Retzius neurons plated on ganglion capsules, the Tshaped pattern of AP cells is simple, well organized, and interestingly, it is also similar to the outgrowth pattern developed by em- 
bryonic AP neurons at around day 9 (17). This similarity between growth of embryonic and adult cultured neurons suggests that an ECM similar to that in the ganglion capsules may provide the initial messages for structuring the outgrowth pattern. This pattern can be quantitatively reproduced if AP neurons are plated on CNS or capsule homogenates as substrates (Figure 2), thus providing the possibility to analyze the steps of its formation. Time-lapse analysis of AP neurons growing on CNS homogenates has shown a complex dynamics of neurite elongation and retraction. As shown in Figure $2 \mathrm{~B}$, while two main neurites grow in opposite directions, secondary and presumably supernumerary neurites are retracted, reflecting competition. Indeed, after a total neuritic length of about $100 \mu \mathrm{m}$, growth of primary neurites occurs at the same rate as retraction of secondary neurites, suggesting an economical mechanism for selective growth plus
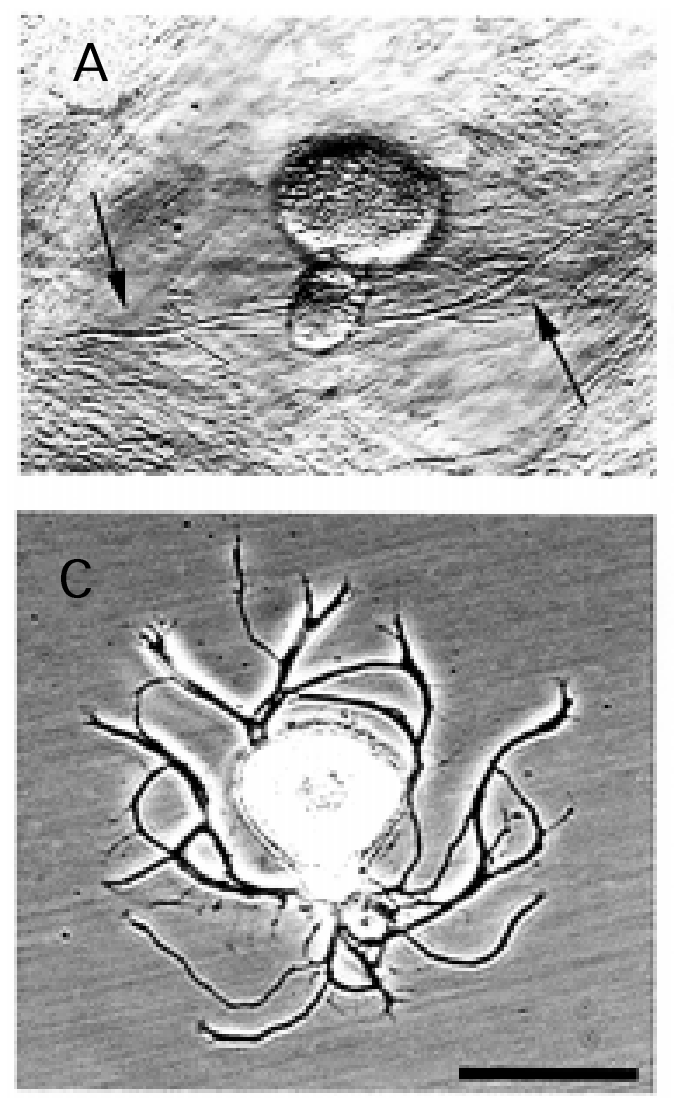

elimination of supernumerary neurites, reutilizing the same precursors (De Miguel FF and Vargas J, unpublished data).

ECM proteins may regulate neurite extension and retraction through a complex set of indirect mechanisms. For example, growth of neurites on leech laminin increases calcium currents evoked by electrical activity in cultured leech neurons (18). In addition, calcium injection into the cells in response to electrical activity may induce neuritic retraction in leech neurons $(19,20)$. Similar events have been described in different neuronal types during development, for example in amphibian spinal neurons in vitro, increases in the intracellular concentration of calcium reduce neurite extension and produce retraction $(21,22)$. Growth cones of leech neurons collapse in response to electrical stimulation $(19,20)$ or to the application of a calcium ionophore, presumably due to a disruption of microfilaments (23). Although
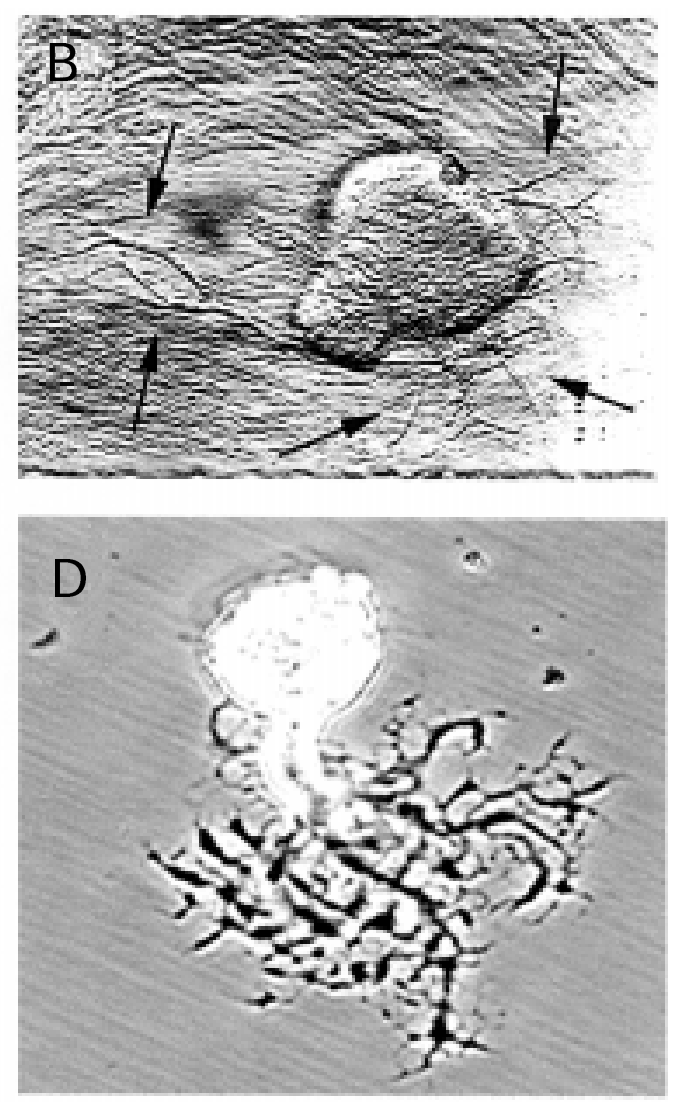

Figure 1 - Outgrowth patterns of anterior pagoda (AP) (A) and Retzius (B) neurons on ganglion capsules. While AP neurons developed two neurites in opposite directions, Retzius neurons developed a different outgrowth pattern with multiple neurites. $C, A P$ neuron growing on leech laminin extract. Multiple long and thin branches were characteristic. D, AP neurons growing on Con A. Scale bar $=30 \mu \mathrm{m}$, same for all neurons (adapted from Ref. 16). 
Figure 2 - Development of the outgrowth pattern of one anterior pagoda (AP) neuron plated on leech CNS homogenate. A, At $48 \mathrm{~h}$ in culture, one branch had grown with several secondary processes. Twenty-four hours later the primary branch was reoriented and one of the secondary branches had elongated (marked by the arrow). At $84 \mathrm{~h}$ in culture, a second branch had grown (marked by the arrow). Both branches continued growing for $24 \mathrm{~h}$. Scale bar $=50$ $\mu \mathrm{m}$ (adapted from Ref. 16). B, Time-lapse analysis of neurite extension and retraction of neurites of the same AP cell presented in A. The time represents the growth time. The large arrows point at neurites that retracted while the small arrows point at neurites that elongated. Scale bar $=40 \mu \mathrm{m}$. the soma of adult AP neurons is unable to produce action potentials (24) these are produced at distant sites. Therefore, it is possible that during neurite extension acti- vation of calcium channels related to electrical activity may also participate in the regulation of growth and retraction of neurites.
A
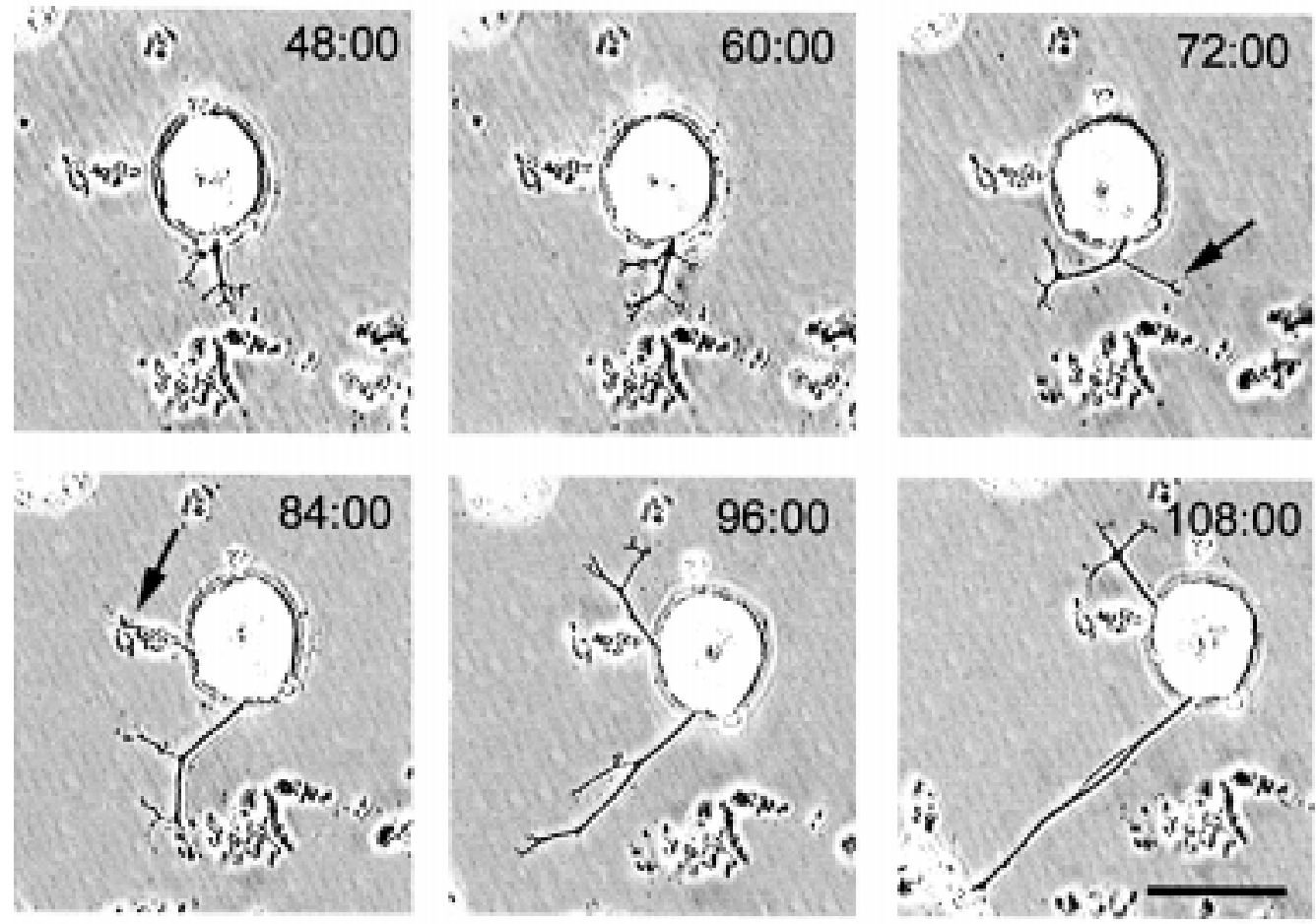

B
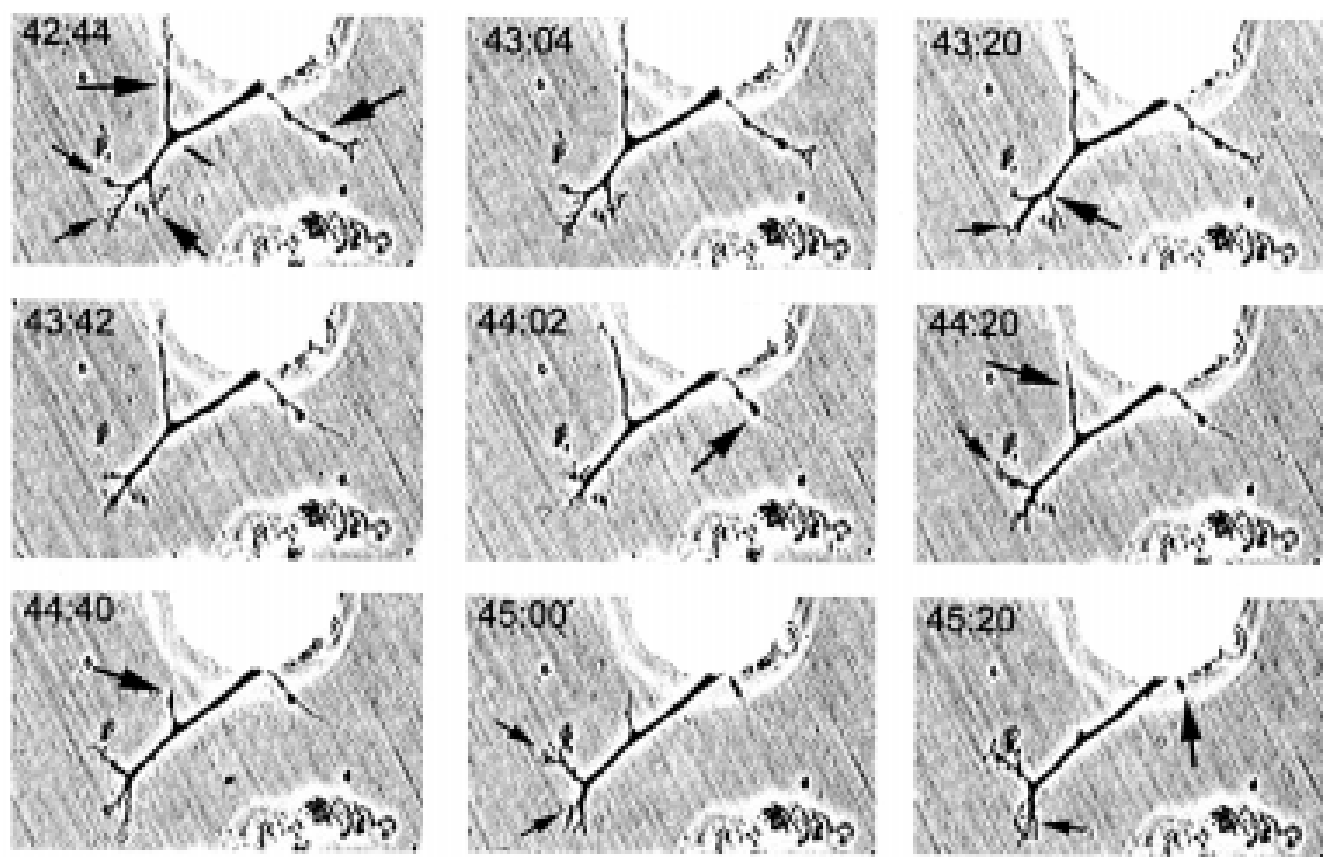


\section{Directing growth}

In addition to influencing neuritic outgrowth, the substrate is also determinant for target finding. When pairs of Retzius neurons are plated at a distance on a ganglion capsule, neurites are sent towards each other and make contact even if the cells were originally plated orienting their processes $180^{\circ}$ from each other. Similarly, ECM on ganglion capsules induces directed growth in pairs of AP neurons. In contrast, directed growth failed when mixed pairs of one Retzius cell and one AP cell were plated on capsules, or when pairs of neurons were plated on Con A or leech laminin. It is noteworthy that in the animal, pairs of Retzius or AP neurons form connections, but Retzius neurons do not contact AP neurons. Therefore, directed growth on capsules again seems to reproduce a developmental phenomenon selectively induced by native ECM proteins. That directed growth fails in pairs of neurons plated on laminin or Con A underscores the importance of ECM inducing multiple effects, such as the possible release of soluble attractants.

\section{Selecting the synaptic attributes}

After finding an appropriate target, a next step in development is the formation of correct synapses. How does a neuron choose when and with what to make a chemical or an electrical synapse? Are neurite outgrowth, directed growth and synapse formation part of a cascade of events triggered by contact with an appropriate substrate? To what extent extrinsic influences may determine neuronal connectivity? An answer to these questions has again been provided by the analysis of growth and synapse formation of leech neurons on different substrates. In the leech, a monosynaptic reflex response that shortens the animal is mediated by the activation of nociceptive $(\mathrm{N})$ sensory neurons that in the ganglion make chemical connection with annulus erector (AE) motoneurons (25). When these two neuronal types are isolated and plated in culture they form an electrical synapse instead of the chemical one (26). The difference between the connection in the animal and in culture provided an attractive preparation to examine if the selection of a synapse is also influenced by the substrate. When growth was tested, $\mathrm{N}$ and $\mathrm{AE}$ neurons had opposite responses to the substrates. As shown in Figure 3, single N cells failed to grow on Con A, but grew profusely on ganglion capsules. In contrast, AE neurons sprouted profusely on Con A but failed to grow on ganglion capsules. Interestingly, as shown in Figure 4, pairs of $\mathrm{N}$ and $\mathrm{AE}$ neurons plated at a distance on the same capsule grew towards each other and made contact. Nevertheless, neurons did not form a chemical synapse but rather developed an electrical rectifying synapse (Figure 4C) similar to that formed on Con A (27). These data provide an example that neurite outgrowth and target recognition may obey different signaling patterns than synapse formation, and suggest the contribution of multiple influences being involved in every step of neuronal development and regeneration. A question that still remains to be answered is what are the signals that induce the formation of the correct chemical synapse during development?

Synapse formation can also be achieved by plating neurons in close apposition to each other. In these conditions, pairs of leech neurons can reform chemical or electrical synapses depending on their identities and on the region of the neuron that makes contact. The characteristics of the electrical synapses formed by Retzius neurons are highly reproducible in culture, regardless of the target. Pairs of Retzius cells form non-rectifying electrical synapses (i.e., current can spread equally well in both directions) in the animal as well as in culture $(6,10)$. In contrast, pressure sensory $(\mathrm{P})$ cells form rectifying electrical connections in which depolar- 
izing current spreads only from the $\mathrm{P}$ cell to its partner, while hyperpolarizing current spreads only in the opposite direction $(1,4)$. From these observations one can conclude that the characteristic synapses established by a particular cell type are, at least in part, determined by their identities. What kind of synapse will a Retzius and a P cell form if plated together? Even though Retzius and P cells form electrical synapses with different targets in culture, when plated together they form purely chemical inhibitory synapses, with the Retzius cell always being presynaptic (11).

In the nervous system, one single neuron

Figure 3 - Outgrowth patterns of nociceptive $(\mathrm{N})$ sensory neurons and annulus erector (AE) motoneurons on ganglion capsules and Con A after $48 \mathrm{~h}$ in culture. $\mathrm{A}, \mathrm{N}$ sensory neurons on ganglion capsules sprouted long and fine neurites. Some are pointed by the arrows. B, On Con A, N cells did not grow and only extended short lamellar growth cones with fine filopodia, as pointed by the arrows. C, AE motoneurons plated on ganglion capsules failed to grow. Only fine filopodia elongated from the remaining stump, as pointed by the arrows. D, On Con A, AE motoneurons sprouted profusely. The stumps are indicated by the asterisks (adapted from Ref. 27).

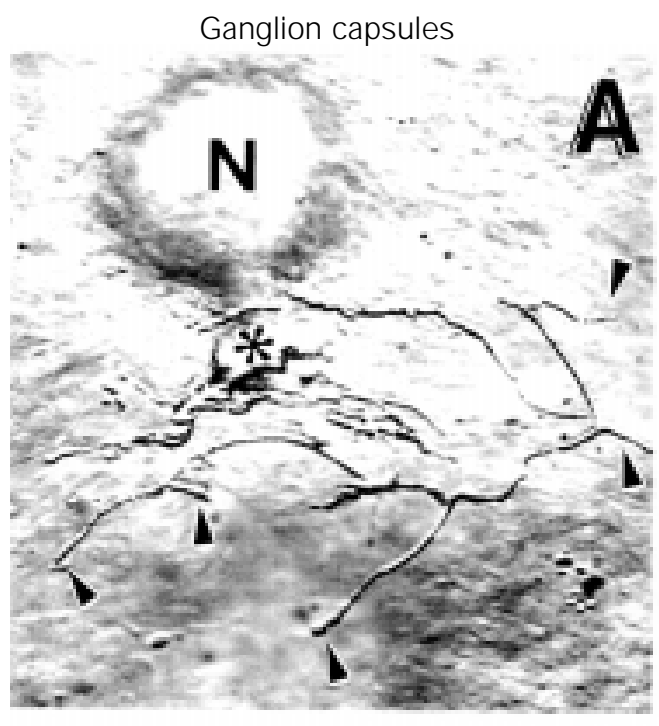

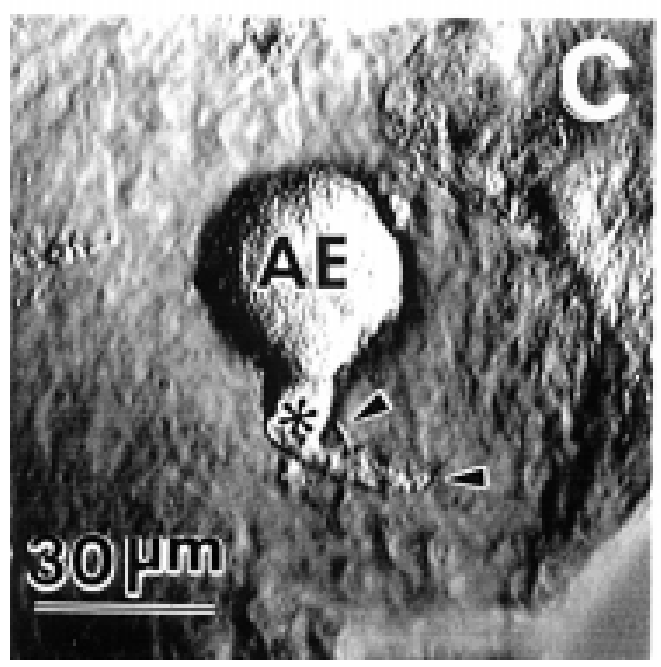
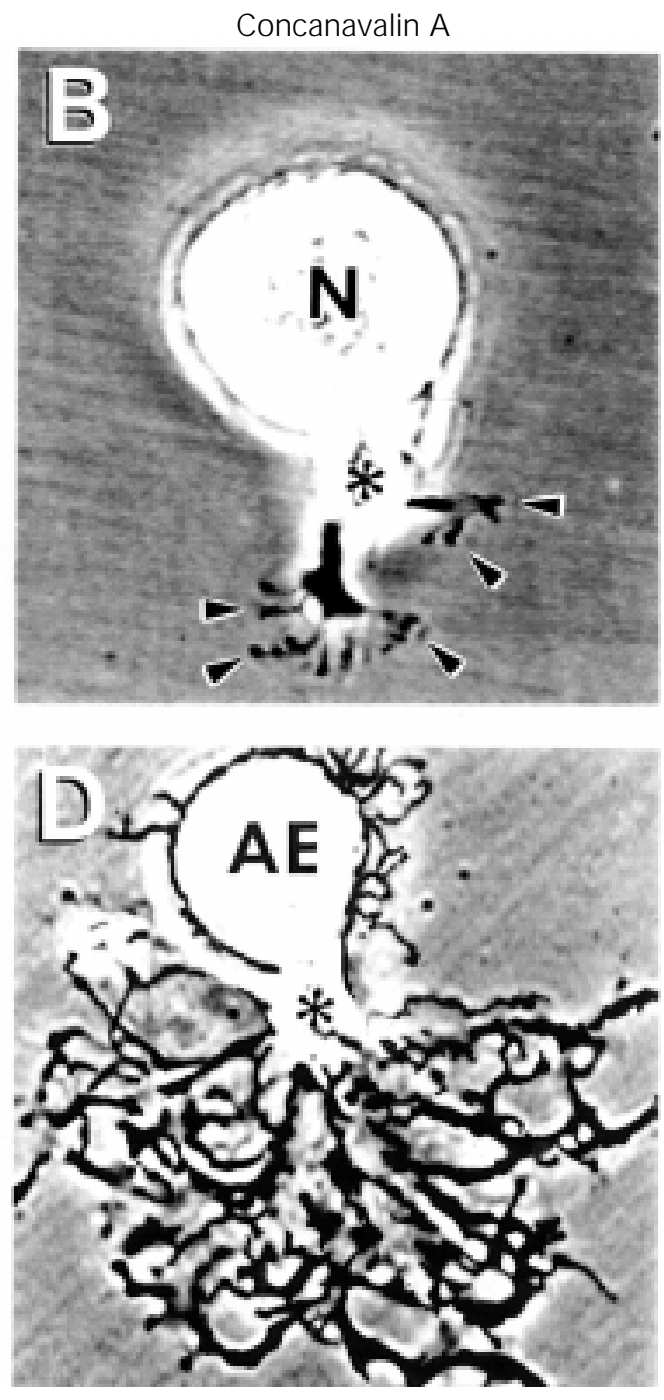

may establish synaptic contacts with many other neurons. Chemical synapses may be excitatory or inhibitory; other synapses may be electrical, either rectifying or non-rectifying. For each particular case, different membrane specializations are required. To what extent are different regions of the cell membrane pre-appointed to form chemical, electrical, pre- or postsynaptic specializations? Liu and Nicholls (10), plating pairs of Retzius neurons with different membrane regions making contact, have provided interesting information. Particular membrane segments of leech neurons have shown different preferences to form particular synaptic modali- 
ties. The tip of the stump, a remaining portion of the primary process, is a preferential region from which Retzius neurons can form presynaptic chemical endings. In contrast, the soma of a Retzius cell forms postsynaptic but never presynaptic components. In addition, either region develops electrical synapses after one or two days.

\section{Modifications during synapse forma- tion: changes in release sites and calcium current distribution}

The series of events that start with neuronal contact continue long after synapses have formed and induce permanent changes in pre- and postsynaptic neurons. Synapse formation requires a whole set of adaptations, including the incorporation of presynaptic vesicles and ion channels in presynaptic terminals and transmitter receptors on the postsynaptic side, among many other components. For example, calcium channels should cluster at the presynaptic endings to regulate transmitter release, while transmitter receptors should be located at the postsynaptic endings $(4,5)$. Cultured serotonergic synapses between two Retzius neurons or between a Retzius and a P neuron have also been useful to analyze the consequences of synapse formation in the incorporation of presynaptic terminals at the sites of contact

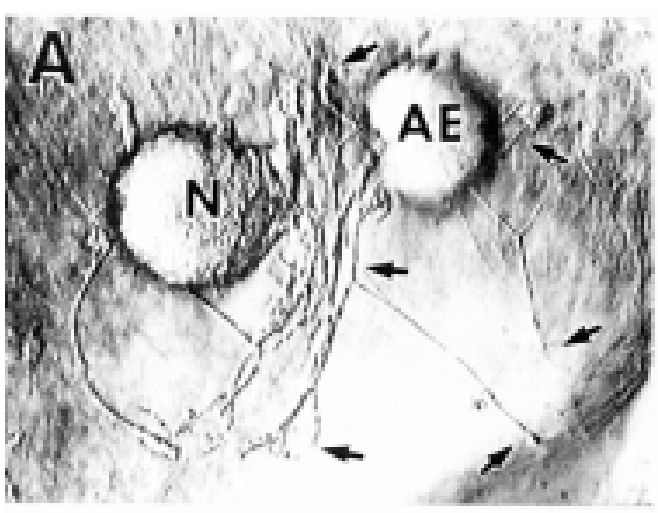

C
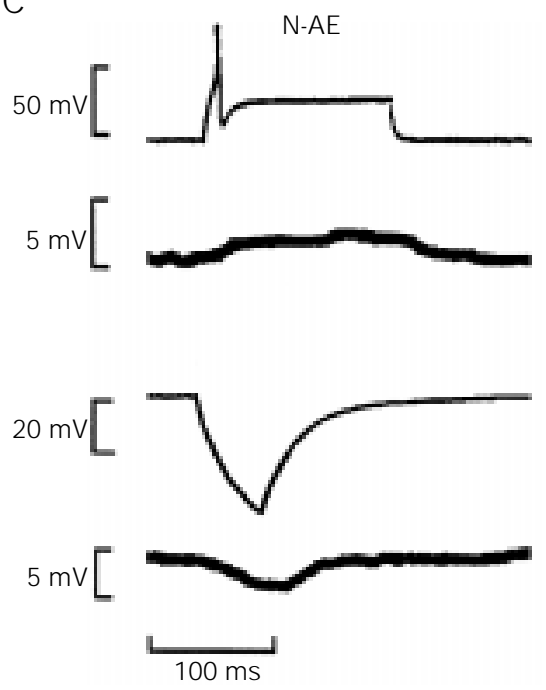

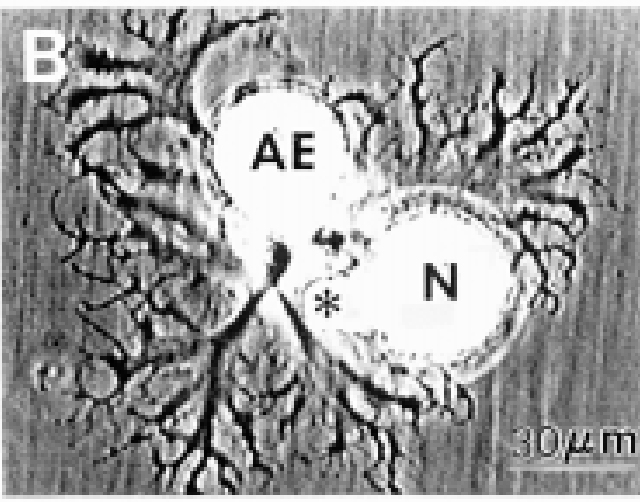

AE-N
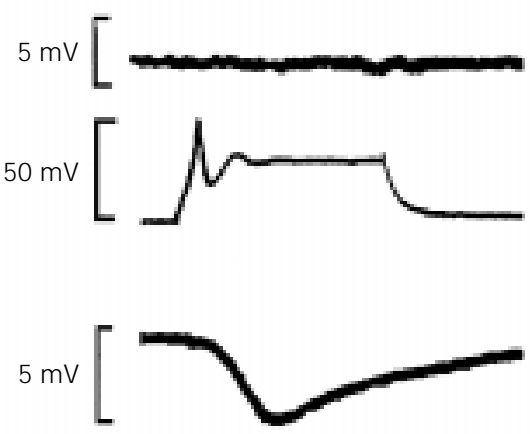

$20 \mathrm{mV}$

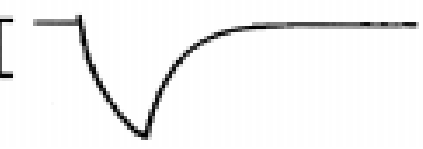

Figure 4 - Growth and synapse formation of pairs of nociceptive $(\mathrm{N})$ and annulus erector (AE) neurons. A, Nomarski image of a pair of neurons growing on a ganglion capsule. In this case both neurons extended processes that made contact. Arrows point at processes growing from the $A E$ neuron. $B$, Only $A E$ neurons sprouted on Con $A$. This phase contrast image shows processes in dark tones. The stump of the $\mathrm{N}$ cell is marked by the asterisk. As can be seen, no neurites are emerging from this stump. C, Rectifying electrical synapse between $\mathrm{N}$ and $\mathrm{AE}$ neurons after $48 \mathrm{~h}$ plated on ganglion capsules. In all cases, traces from the $\mathrm{N}$ cell are above and those from the $\mathrm{AE}$ neuron are below. Low gain is used for the cell in which current is passed and high gain is used for the follower cell. Depolarization spreads only from $\mathrm{N}$ to $A E$ neuron (left-hand traces), while hyperpolarization spreads in both directions. The characteristics of this coupling remained after nine days in culture without traces of a chemical component (adapted from Ref. 27). 
Figure 5 - A, Pair of Retzius and pressure sensory $(P)$ cells forming a chemical synapse. Retzius neurons become always presynaptic to $P$ cells. Scale bar $=50$ $\mu \mathrm{m}$. B and C, Fluorescent staining of presynaptic release sites with the dye FM1-43. In B, the tip of the stump of the presynaptic Retzius neuron develops release sites a few hours after plating in contact with a $\mathrm{P}$ neuron (pointed by the arrow). These terminals are absent in neurons that have not made contact, as can be seen in a single Retzius neuron shown in $C$. Scale bars in $B$ and $C=30 \mu \mathrm{m}$. D, Simultaneous recordings from Retzius and $\mathrm{P}$ neurons. Action potentials in Retzius neurons were produced by current injection through the microelectrode. A single action potential in a Retzius neuron produces a synaptic potential in the $P$ neuron. The synaptic potentials presented here are reversed by the injection of chloride through the intracellular electrode. Pairs of impulses to the presynaptic neuron can elicit facilitation of transmitter release. Two impulses separated by 300 ms produce similar synaptic potentials. In contrast, shortening the delay between impulses produces marked facilitation (Trueta $\mathrm{C}$ and De Miguel FF, unpublished results).
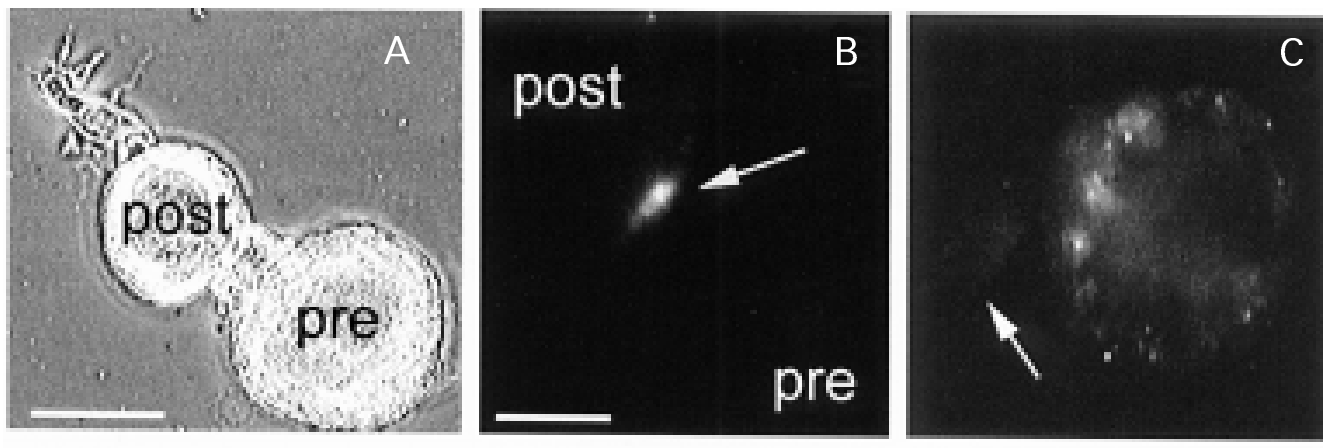

D
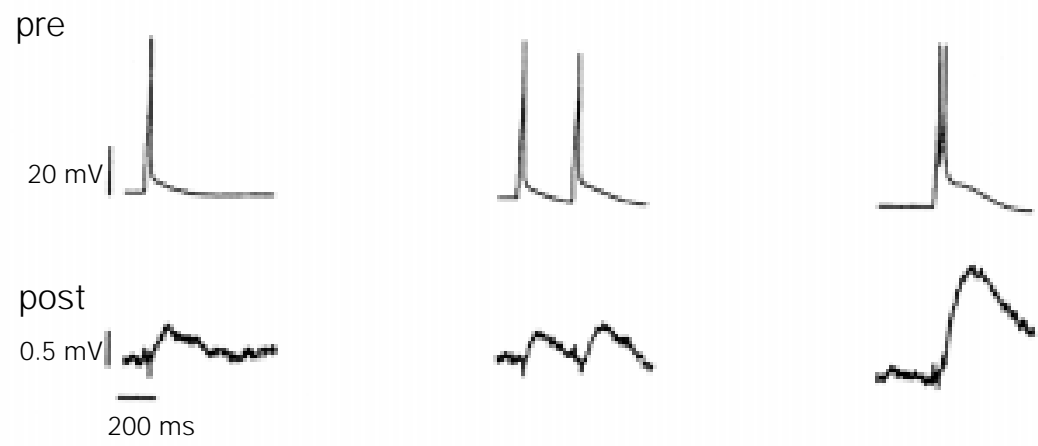

or in the distribution of calcium currents.

The stump of a Retzius neuron forms presynaptic endings on a $\mathrm{P}$ neuron or on another Retzius neuron, with a rapid accumulation of newly produced release sites (Hernandez H, Trueta C and De Miguel FF, unpublished results). Figure 5 shows a pair of Retzius and $\mathrm{P}$ neurons making contact. Terminals shown in Figure 5B and $\mathrm{C}$ have been stained with the fluorescent dye FM143 which is taken up by synaptic vesicles upon endocytosis. Release sites shown in Figure 5B develop within a few hours right at the contact site of the presynaptic Retzius neuron. In single Retzius neurons, release sites are virtually absent from the stump of the cell. Upon synapse formation, electrical activity such as that in Figure 5D can be recorded. An action potential in a Retzius neuron produces a synaptic potential in the $P$ neuron. In this case, the synaptic potentials have been artificially reversed by the injection of chloride through the intracellular elec- trode. The figure also shows that when pairs of impulses are produced close to each other, the synaptic potential shows facilitation, a typical property of chemical synapses (Trueta $\mathrm{C}$ and De Miguel FF, unpublished results).

An ultrastructural study of Retzius neurons has shown clouds of dense-cored vesicles surrounded by clear-cored vesicles in the cytoplasm of the neuronal stump, near to but not in contact with the presynaptic membrane, suggesting that assemblies of presynaptic elements are a step preceding the formation of release sites. Figure 6 shows a typical presynaptic ending of a Retzius neuron with clear vesicles near the presynaptic membrane and surrounded by densecored vesicles (28). Some of the clear vesicles are in close apposition with the membrane. Figure 6B and $\mathrm{C}$ shows two serial sections of a bouton-like structure in which dense-cored vesicles are surrounding clear-cored vesicles. There is no evidence of contact of vesicles with the presynaptic membrane (Hernandez 
Figure 6 - A, Morphology of a characteristic presynaptic ending in a Retzius neuron. Dense-cored vesicles surround clear vesicles near the cell membrane. B and C are two serial sections of a Retzius neuron showing intracellular "clouds" of clear vesicles surrounded by dense-cored vesicles but without making contact with the cell membrane. Presumably this accumulation of vesicles could be a step preceding the formation of a presynaptic ending (Hernandez $\mathrm{H}$, Morales $\mathrm{M}$ and De Miguel FF, unpublished results). Scale bar $=200 \mathrm{~nm}$.

$\mathrm{H}$, Morales $\mathrm{M}$ and De Miguel FF, unpublished results).

The establishment of chemical synapses also correlates with a redistribution of calcium currents (29). The large size of Retzius neurons and the possibility that they have access to very near sites and to the presynaptic endings allowed us to use the loose-patch clamp technique (30) to record local macroscopic currents from different membrane areas of Retzius neurons, thus providing a map of current density. The technique consists of having a large tip-diameter pipette (5 $\mu \mathrm{m})$ and establishing a low resistance seal with the membrane. In this way, ion currents inside the pipette can be isolated and the pipette can be removed and introduced at another location without damaging the cell membrane. Since the same pipette is used over and over again to record from different membrane areas, the amplitude of the currents at different locations provides a map of current density. Using cultured synapses between pairs of Retzius neurons allowed us to compare currents between pre- and postsynaptic neurons of the same pair.

Formation of chemical synapses induces a marked reduction in postsynaptic calcium currents at the cell stump without affecting currents in other cell regions. In contrast, the characteristic amplitudes of calcium currents along presynaptic cells remain unaffected (29). Interestingly, as can be seen in Figure 7 , synapse formation also has a retrograde effect on calcium currents. In triplets of cells in which one cell is purely presynaptic, the middle one is both pre- and postsynaptic and
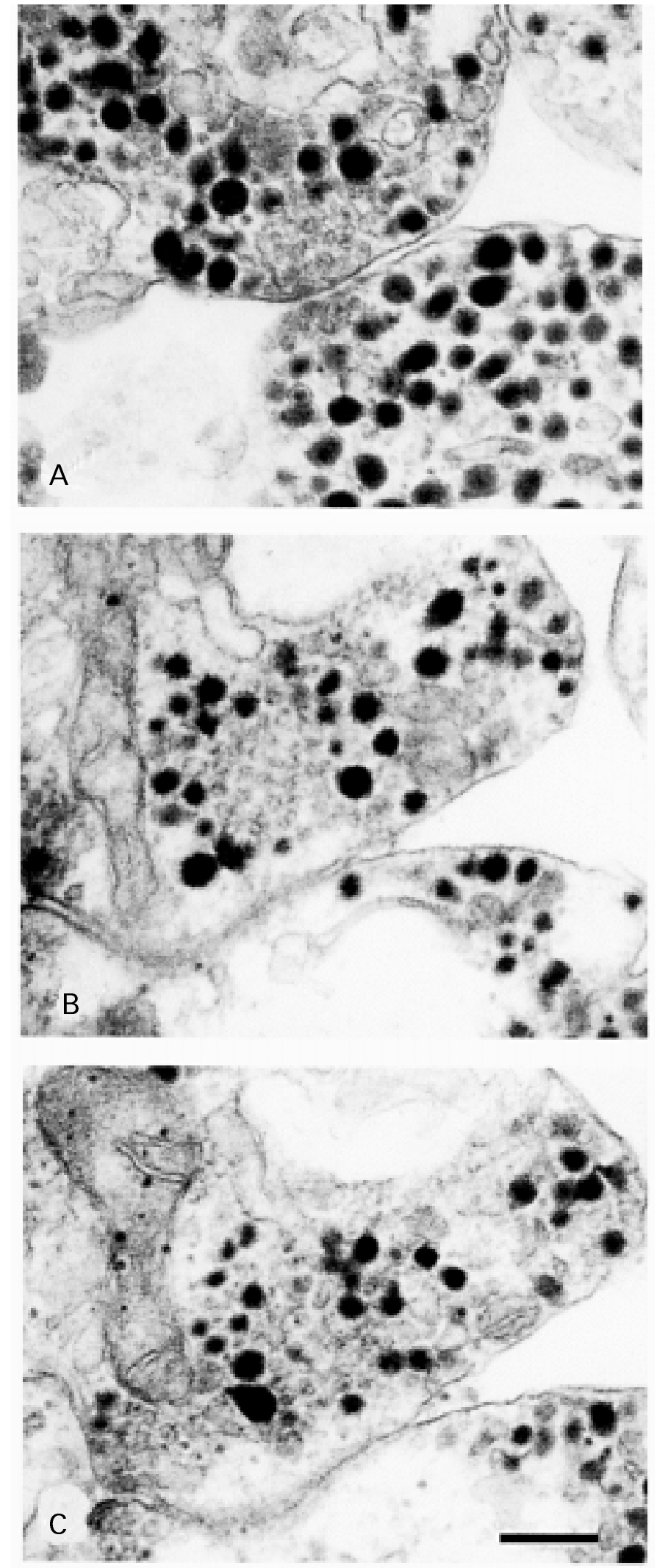
Figure 7 - Anterograde and retrograde effects of synapse formation on calcium currents in Retzius $(R)$ cells. The amplitude of calcium tail currents recorded on different spots of the neuronal membrane reflects the distribution of voltage-sensitive channels. A, Amplitudes of tail currents in different locations of pre- and postsynaptic cells. The cell on the top is presynaptic and the cell on the bottom is postsynaptic. The current at the stump of the postsynaptic cell is markedly decreased relative to the same location on the presynaptic cell. Currents in presynaptic cells and in the soma of postsynaptic cells remain unchanged when compared to single cells. $\mathrm{B}, \mathrm{A}$ retrograde signal can also be detected in triplets of cells. Only currents at the stumps are shown, since the somatic currents did not change. The cell in the middle (postsynaptic to the one on the top but presynaptic to the one on the bottom) does not decrease its calcium current at the stump, although it is postsynaptic to the cell on the top (adapted from Refs. 29,31).
A

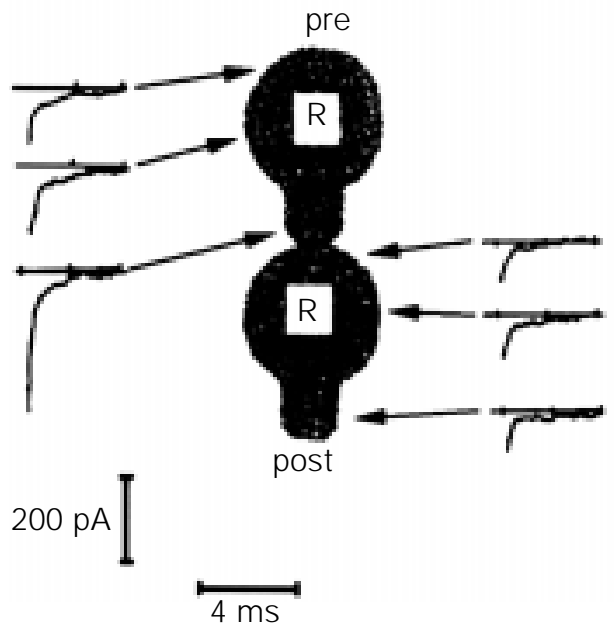

B

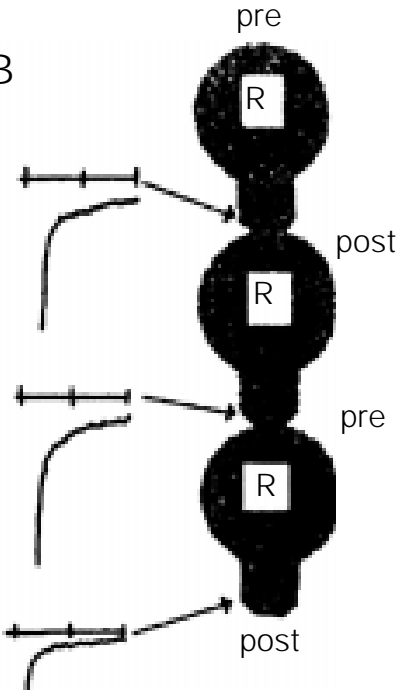

the third cell is purely postsynaptic. Calcium currents are reduced only in the purely postsynaptic cell, while the cell in the middle is unaffected even though it has also postsynaptic elements. In addition, neurite outgrowth of the postsynaptic neuron is inhibited by about $70 \%$ (31). Anterograde influences have been detected by Drapeau and his colleagues $(4,5)$, affecting the postsynaptic responses to transmitter in P cells, in which contact with Retzius neurons induces the loss of an extrasynaptic receptor to serotonin.

\section{Conclusions and future directions}

From studies with identified leech neurons in culture, it has been possible to understand some of the events underlying neurite outgrowth and synapse formation. In addition, the well-controlled conditions of the culture system have provided the chance to analyze the mechanisms of transmitter release in great detail (1). An interesting future direction will be to analyze the molecular basis for these signals. Approaches such as production of DNA libraries and injection of cloned RNA or DNA are currently being used by Blackshaw and her group (32) in studies on these large neurons. Another avenue that has only begun to be explored is the relationship between neurite outgrowth, the molecules which support it and the specificity of synapse formation. Ultimately, the mechanisms characterized with adult neurons in culture need to be tested with these same neurons in the developing CNS. Thus, the CNS of the leech offers the rare possibility of characterizing signaling mechanisms during synapse formation and neurotransmission, and of determining the roles of these mechanisms during in vivo development and regeneration.

\section{References}

1. Nicholls J G (1987). The search for connections: Studies of regeneration in the nervous system of the leech. Magnes Lecture Series. Vol. II. Sinauer Associates Inc., Sunderland, MA

2. Kater SB \& Mills RL (1991). Regulation of growth cone behavior by calcium. J ournal of Neuroscience, 11: 891-899.

3. Dagan D \& Levitan IB (1981). Isolated identified Aplysia neurons in cell culture. J ournal of Neuroscience, 7: 736-740.

4. Fernández-de-Miguel $F \&$ Drapeau $P$ (1995). Synapse formation and function: Insights from identified leech neurons in culture. J ournal of Neurobiology, 27: 367379.

5. Haydon PG \& Drapeau P (1995). From contact to connection: Early events during synaptogenesis. Trends in Neurosciences, 18: 196-201.

6. Ready DF \& Nicholls J (1979). Identified 
neurones isolated from the leech CNS make selective connections in culture. $\mathrm{Na}$ ture, 281: 67-69.

7. Masuda-Nakagawa LM \& Wiedemann C (1992). The role of matrix molecules in regeneration of leech CNS. J ournal of Neurobiology, 23: 551-567.

8. Von Bernhardi R \& Muller KJ (1995). Repair of the central nervous system: lessons from lesions in leeches. J ournal of Neurobiology, 27: 353-366.

9. Dietzel ID, Drapeau P \& Nicholls J G (1986). Voltage dependence of 5-hydroxytryptamine release at a synapse between identified leech neurones in culture. J ournal of Physiology, 372: 191-205.

10. Liu Y \& Nicholls J G (1989). Steps in the development of chemical and electrical synapses by pairs of identified leech neurones in culture. Proceedings of the Royal Society of London. B, Biological Sciences, 236: 253-268.

11. Fuchs PA, Nicholls J G \& Ready DF (1981). Membrane properties and selective connections of identified leech neurones in culture. J ournal of Physiology, 316: 203223.

12. Bixby J L \& Harris WA (1991). Molecular mechanisms of axon growth and guidance. Annual Review of Cell Biology, 7: 117-159.

13. Sanes J R (1989). Extracellular matrix molecules that influence neural development. Annual Review of Neuroscience, 12: 491516.

14. Grumbacher-Reinert S (1989). Local influence of substrate molecules in determining distinctive growth patterns of identified neurons in culture. Proceedings of the National Academy of Sciences, USA, 86: 7270-7274.

15. Fernandez de Miguel $F$ (1997). Outgrowth patterns and directed growth of identified neurons induced by native substrates in culture. J ournal of Comparative Neurology, 380: 1-15.

16. De-Miguel FF \& Vargas J (1999). Extracellular matrix induces a well-organized outgrowth pattern with neurite extension and retraction in cultured leech neurons. J ournal of Comparative Neurology, 417: 387-398.

17. Gao WQ \& Macagno ER (1987). Extension and retraction of axonal projections by some developing neurons in the leech depend upon the existence of neighboring homologues. II. The AP and AE cells. J ournal of Neurobiology, 18: 295-313.

18. Ross WN, Arechiga $\mathrm{H} \&$ Nicholls JG (1987). Influence of substrate on the distribution of calcium channels in identified leech neurons in culture. Proceedings of the National Academy of Sciences, USA, 85: 4075-4078.

19. Grumbacher-Reinert $S \&$ Nicholls JG (1992). Influence of substrate on retraction of neurites following electrical activity of leech Retzius cells in culture. J ournal of Experimental Biology, 167: 1-14.

20. Neely MD (1993). Role of substrate and calcium in neurite retraction of leech neurons following depolarization. J ournal of Neuroscience, 13: 1292-1301.

21. Holliday J \& Spitzer NC (1990). Spontaneous calcium influx and its roles in differentiation of spinal neurons in culture. Developmental Biology, 141: 13-23.

22. Gomez TM \& Spitzer NC (1999). In vivo regulation of axon extension and pathfinding by growth cone-calcium transients. Nature, 397: 350-355.

23. Neely D \& Gesemann M (1994). Disruption of microfilaments in growth cones following depolarization and calcium influx. J ournal of Neuroscience, 14: 75117520.

24. Melinek R \& Muller KJ (1996). Action potential initiation site depends on neu- ronal excitation. J ournal of Neuroscience, 16: 2585-2591.

25. Nicholls J G \& Purves D (1970). Monosynaptic chemical and electrical connections between sensory and motor cells in the central nervous system of the leech. J ournal of Physiology, 209: 647-667.

26. Vyklicky L \& Nicholls J G (1988). Specificity of connections formed by nociceptive cells of the leech in tissue culture. J oumal of Experimental Biology, 134: 17-26.

27. De Miguel FF \& Vargas J (1997). Different determinants on growth and synapse formation in cultured neurons. NeuroReport, 8: 761-765.

28. Kuffler DP, Nicholls JG \& Drapeau P (1987). Transmitter localization and vesicle turnover at a serotoninergic synapse between identified leech neurons in culture. J ournal of Comparative Neurology, 256: 516-526.

29. Fernández-de-Miguel $\mathrm{F}$, Cooper $\mathrm{R} \&$ Adams WB (1992). Synaptogenesis and changes in calcium current distribution in cultured leech cells. Proceedings of the Royal Society of London. B, Biological Sciences, 247: 215-221.

30. Stühmer W, Roberts WS \& Almers W (1983). The loose patch clamp. In: Sakmann B \& Neher E (Editors), Single Channel Recording. Plenum Press, New York, London, 123-132.

31. Cooper RL, Fernández-de-Miguel $F$, Adams WB \& Nicholls J G (1992). Anterograde and retrograde effects of synapse formation on calcium currents and neurite outgrowth in cultured leech neurons. Proceedings of the Royal Society of London. B, Biological Sciences, 249: 217-222.

32. Korneev S, Blackshaw $S \&$ Davies JA (1994). cDNA libraries from a few neural cells. Progress in Neurobiology, 42: 339346. 